Review

\title{
Effectiveness of Aromatherapy in Alleviating Palliative Symptoms and Its Possible Side Effects: A Literature Review
}

\author{
Heidrun Männle *, Birthe Osorio, Karsten Münstedt
}

Ortenau Klinikum Offenburg-Kehl, Ebertplatz 12, 77654 Offenburg, Germany; E-Mails: heidrun.maennle@ortnau-klinikum.de; birthe.osorio@ortenau-klinikum.de; karsten.muenstedt@ortenau-klinkum.de

* Correspondence: Heidrun Männle; E-Mail: heidrun.maennle@ortnau-klinikum.de

Academic Editor: Leila Kozak

Special Issue: Integrative Therapies in Palliative Care

OBM Integrative and Complementary Medicine

2020 , volume 5 , issue 2

doi:10.21926/obm.icm.2002024
Received: March 09, 2020

Accepted: April 16, 2020

Published: April 28, 2020

\begin{abstract}
Palliative care is a steadily growing component of medicine that focuses on treating individuals with life-limiting diseases such as cancer. It aims at improving the quality of life and alleviating cancer-related symptoms, including anxiety, pain, dyspnea, cough, nausea/vomiting, and constipation, which could result in significant physical and mental impairment. Integrative palliative care (IPC), as a subspecialty of palliative care, includes complementary approaches to treat patients holistically in their biopsychosocial environments. Aromatherapy is a complementary therapy widely used in patients with cancer. It is practiced in two forms: direct inhalation of essential oils and in the form of massage with essential oils. The present study investigated the effectiveness of aromatherapy in alleviating palliative symptoms and its possible side effects.
\end{abstract}

\section{Keywords}

Aromatherapy; palliative care; essential oils

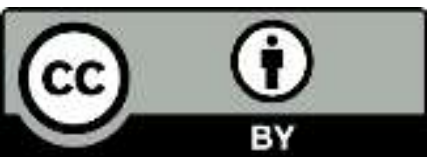

(C) 2020 by the author. This is an open access article distributed under the conditions of the Creative Commons by Attribution License, which permits unrestricted use, distribution, and reproduction in any medium or format, provided the original work is correctly cited. 


\section{Introduction}

The incidence rates of cancer have been rapidly increasing worldwide. According to GLOBOCAN 2018, an online database that provides incidence and mortality rates of 36 types of cancers, 18.1 million new cases of cancer and 9.6 million deaths from cancer were reported in 2018 [1]. This increasing burden is accompanied by an increase in the number of patients with cancer who suffer from cancer-related complaints and problems. In this regard, palliative care focuses on the treatment of people with life-limiting diseases to improve their quality of life and reduce adverse effects.

Complementary and alternative medicine (CAM) has emerged as an important aspect of palliative care. A survey in a palliative setting revealed that $40 \%$ of patients used CAM [2], with a preference for biological approaches such as trace elements (64\%), vitamins (60\%), other supplements (60\%), and medical herbs (64\%) [2]. Aromatherapy, a widely and often used complementary therapy in patients with symptoms requiring palliative care, is the use of scents to incite psychological or physiological responses for treatment. Medical aromatherapy uses selected essential oils (EOs), which are concentrated natural plant products containing volatile aroma compounds. The majority of EOs are derived by steam distillation, a tradition nearly 5,000 years old. EOs have several potential applications owing to their different properties. They are mostly used as direct inhalers, in the form of massage, or as an ointment. Hundreds of receptor cells in the nose relay the smell to the brain via the olfactory nerve, thereby acting on organ systems, blood circulation, and pain processing pathways [3].

One of the aspects to be considered while using aromatherapy as a "gentle therapy" in palliative care is that its effectiveness must be proven based on evidence to rule out serious side effects. However, any effective medication may also have side effects. Consequently, the present study summarizes the usefulness of aromatherapy in treating symptoms that require palliative care and its possible side effects. To answer this question, a literature review on this topic was performed using PubMed.

\section{Materials and Methods}

\subsection{Literature Search}

A systematic literature search was conducted using PubMed (Medline) according to the PRISMA (Preferred Reporting Items for Systematic Reviews and Meta-Analyses) guidelines on January 11, 2020. The following keywords were used: "aromatherapy" AND "palliative care" and the symptom in question. Symptoms included pain, nausea and vomiting, constipation, anxiety, dyspnea, cough, fatigue, and insomnia. An additional search using the terms "essential oils" AND "palliative care" was conducted.

In the second step, we analyzed the references in all retrieved publications, including metaanalyses and systematic reviews, to further identify the publications that might have been missed in the referred databases.

\subsection{Criteria for Eligibility}

All publications from the past 25 years identified in the first two steps were included if their full- 
texts could be retrieved in English and German.

\subsection{Selection of Publications}

Titles and abstracts of retrieved publications were checked by two authors of the study (H.M., K.M.) for their fulfillment of the eligibility criteria. Publications that did not meet the criteria for eligibility, as well as duplicate publications, were excluded from the study. Finally, the full texts of the included publications were analyzed.

\section{Results}

Literature Search:

Pain: The initial search strategy yielded 28 titles. Most items were excluded due to misleading titles. Eight articles that met the eligibility criteria were read and reviewed completely. Three more articles were excluded because they did not specifically measure the pain symptom; similarly, another article was excluded as it did not include palliative care. Finally, four articles were included in the study.

Nausea and Vomiting: The initial search strategy yielded seven titles. Most items were excluded owing to duplicate records or misleading titles. Five articles that met the eligibility criteria were read and reviewed completely. Three more were excluded as these did not specifically record nausea and vomiting. Finally, two articles were included in the study.

Constipation: The initial search strategy yielded two titles. Both titles were completely read and included in the study.

Anxiety: The initial search strategy yielded 26 titles. Most items were excluded owing to misleading titles. Seventeen articles that met the eligibility criteria were read completely. Five more articles were excluded.

Depression: The initial search strategy yielded 13 titles. Most items were excluded owing to duplicate records or misleading titles. Finally, three articles were read completely and included in the study.

Fatigue: The initial search strategy yielded eight titles. Most items were excluded owing to duplicate records or misleading titles. Four articles that met the eligibility criteria were read completely. Three more articles were excluded as these did not specifically record fatigue. Finally, one article was included in the study.

Insomnia: The initial search strategy yielded three titles. All were read completely. One article was excluded because it did not specifically record insomnia. The remaining two articles were included in the study.

Cough: The initial search strategy yielded one title; however, it was excluded because it did not specifically investigate cough.

Dyspnea: The initial search strategy yielded one title, which was excluded as it did not specifically investigate cough. The additional search using the terms "essential oils" AND "palliative care" revealed no further relevant studies

\subsection{Pain}

In order to study the effect of aromatherapy on pain, a systematic review of randomized 
controlled trials focusing on two trials involving aromatherapy practiced by inhaling oil and six trials that compared massage with aromatherapy was carried out [4]. However, only one trial measured the long-term effect of aromatherapy on pain and anxiety; it compared a 4-week treatment using massage with lavender essential oil and an inert carrier oil, an inert carrier oil massage only, and no intervention [5]. Forty-two patients were randomly allocated to treatments. For each group, no statistically significant differences regarding pain were observed between the baseline and at the end of the study. The different arms were not compared to each other, and side effects were not reported.

The systematic review indicated that the trials were poorly conducted and did not provide any clear evidence, making it impossible to provide any evidence-based recommendations [4].

Several studies were not included in this review:

- A trial involving 60 pediatric patients compared the use of aromatherapy scented sachets with the use of visual imagery to treat pain. The trial showed an improvement in pain following the aromatherapy intervention [6]. However, this trial also had certain limitations. Children with medical exclusion criteria for aromatherapy, for example, hypersensitive airways, were included in the control arm, which could have created bias.

- A Canadian community hospital developed an integrated complementary therapy pilot program in the palliative care unit. Different therapies included aromatherapy and massage, Reiki, and therapeutic touch. Several aromatherapy products are available to improve pain; for example, aromatherapy massage oil, aromatherapy mouth care solution for malodor or pain in the mouth, and aromatherapy ointment for red, inflamed, and/or painful intact skin. Thirty-one patients were asked to rate the severity of symptoms before and after one or two sessions. The analysis revealed a significant decrease in symptom severity, especially in pain by $44 \%$ [7].

The presented trials in the palliative care setting may not provide concrete evidence in favor of the use of aromatherapy to control pain. However, evidence from meta-analyses and systematic reviews performed in patients undergoing cardiac surgery [8], patients with primary dysmenorrhea [9], and those with pain during labor [10] show that aromatherapy can improve the pain symptom. Another meta-analysis and systematic review reported the successful application of aromatherapy to treat pain when combined with conventional treatments [11].

\subsection{Nausea and Vomiting}

The trial involving pediatric patients also investigated the effectiveness of aromatherapy scented sachets in reducing nausea. Thirty children in each arm of the trial were examined compared with children in the other arm who were shown visual imagery. Improvement in nausea was observed at 5 minutes, which continued until 60 minutes post-exposure $(p<0.001)[6]$.

Another single-arm trial involving 160 patients examined the effects of aroma sticks on anxiety, nausea, and difficulty in sleeping. Forty-five aroma sticks were distributed among the patients for nausea management. Patients were asked to hold the sticks under their noses while inhalation; $77 \%$ of patients reported that they benefitted from the therapy. However, $18 \%$ reported no positive effect, whereas $8 \%$ never used their aroma sticks. Moreover, $82 \%$ of the patients found the aroma stick beneficial for the management of nausea. The effect was related to the frequency of use of aroma sticks; the effect was more pronounced with more frequent use. Patients did not 
report side effects [12].

While limited evidence may be available regarding the effectiveness of aromatherapy in a palliative setting, a systematic review concluded that aromatherapy should be considered as a treatment modality for postoperative nausea and vomiting (PONV) in adult surgical patients [13]. Moreover, it is speculated that it could be used in combination with other interesting complementary approaches, such as ginger and P6 acupressure. For example, it has been proved effective in a systematic review of trials involving pregnant women and, therefore, could be considered a reliable treatment approach [14].

\subsection{Constipation}

A three-armed randomized trial comprising 45 patients with constipation was performed to evaluate the effect of aromatherapy. Here, patients were randomly assigned to three experimental groups: (a) plain abdominal massage, (b) aroma abdominal massage, and (c) control. Massage sessions lasting 15 to 20 minutes were held daily for five consecutive days. The results showed a significant difference in the bowel movements between the aroma massage group and the plain massage group $(p<0.001)$; the aroma massage group and the control group $(p<0.001)$; and the plain massage group and the control group $(p<0.05)$ [15].

Another small, one-arm study comprising 15 patients with constipation was performed; the patients were treated with an aroma massage. In addition, patients were taught to perform the massage themselves. All patients reported a decrease in the discomfort caused by abdominal distension and flatulence after one week of aromatherapy. Eight patients reported recovery to normal bowel function after four to six weeks. Further, three patients reported less severe symptoms associated with constipation; however, they still felt constipated. Four patients were lost to follow-up [16].

Two further trials evaluated the effects of aroma massage on constipation [17-18]. Although the papers were published in Korean, these provided evidence that aroma massage contributed to reduced use of stool softeners, suppositories, and enemas.

\subsection{Anxiety}

A systematic review of randomized controlled trials in 2019 failed to show the effectiveness of aromatherapy massage compared to massage alone [19]. The researchers identified three studies and summarized their results [20-22]. Though Wilkinson et al. demonstrated an enhanced effect of massage with essential oils compared to massage alone [22]. The combined analysis of all trials revealed no significant difference between the intervention (aromatherapy massage) and the control (massage alone) groups [19]. Thus, it could be concluded that the improvement observed in the intervention group (aromatherapy massage) was primarily the result of the massage. In 2007, Wilkinson published a multicenter randomized controlled trial with 288 patients and showed that aromatherapy massage exerted no long-term benefits on anxiety in comparison with usual treatment [23]. From these studies, it could be speculated that restricting the interpretation to randomized trials could create the impression that aromatherapy is not useful.

In a randomized controlled trial by Soden et al., as mentioned above, the researchers measured the effect of aromatherapy on pain and anxiety by comparing a 4-week course of aromatherapy massage with that with lavender essential oil, massage with an inert carrier oil only, and no 
intervention [5]. No statistically significant changes were observed between the baseline and at the end of the study for each group. Moreover, different arms were not compared to each other. The findings of Soden et al. corroborate with those of Hsu et al. [19].

Two studies examined the long-term effects of aromatherapy on anxiety [24-25].One study showed a statistically significant difference between the two trial arms with respect to the effect of aromatherapy on anxiety [24]. Participants in the experimental group were asked to inhale rose water for four weeks, whereas those in the control group did not undergo any intervention. The mean scores of anxiety in the experimental group were significantly reduced $(p<0.001)$. The second trial compared the effectiveness of combining the aromatherapy massage with cognitive behavior therapy to address the symptoms of emotional distress. Both arms showed an improvement in symptoms; however, the between-group comparison showed a non-significant trend toward greater improvement with cognitive behavior therapy [25].

In a Canadian trial by Berger et al. with an integrated complementary therapy pilot program implemented within the palliative care unit, 31 patients were asked to rate the severity of symptoms before and after one or two sessions. Aroma stick inhalers were used to treat breathlessness and/or anxiety. The analysis revealed a significant decrease in anxiety by $66 \%$ [7].

Furthermore, aroma sticks have been used in a trial conducted by Stringer et al. The study comprised 49 patients who considered anxiety as their main concern; out of these, 39 patients found aroma sticks to exert beneficial effects [12].

In a small, single-arm trial, aromatherapy massage was applied to patients with malignant brain tumors. The Hospital Anxiety and Depression Scale (HADS) was used, and the results showed no significant differences before and after the aromatherapy massage. However, patients' subjective perception of relaxation was reported and supported by significantly reduced physical parameters of the autonomic nervous system [26]. In another single-arm trial, aromatherapy massage was offered as a 6-week, 6-treatment course, with 58 patients completing the six sessions. Anxiety, assessed using HADS, was significantly improved; the score increased from 8.9 to $6.2(p<0.001)$ [27].

Overall, some evidence exists that aromatherapy could have a positive influence on anxiety, especially the possible anxiolytic effects of lavender [28]. Systematic reviews and a meta-analysis have shown that critically ill adults and patients undergoing cardiac surgery could benefit from aromatherapy approaches $[8,29]$.

\subsection{Depression}

The same trials that evaluated anxiety also studied depression using $\operatorname{HADS}[5,23,27]$. Soden et al. were unable to show the effectiveness of aromatherapy massage on symptoms of depression. The message alone resulted in statistically significant reduction in depression scores [5]. However, Kite et al. recorded a significant improvement in HADS depression scores, from 6.1 to 4.0 ( $p<$ 0.001 ), in a single-arm trial that offered aromatherapy massage as a 6-week, 6-treatment course [27]. Conversely, the multicenter randomized controlled trial published by Wilkinson et al. showed that aromatherapy massage did not exert long-term benefits on depression, as shown for anxiety [23].

Two systematic reviews, including one with a meta-analysis, have addressed the topic of aromatherapy and depression. One study concluded that aromatherapy could be beneficial in 
attenuating the psychological symptoms, such as anxiety and depression, in postmenopausal and elderly women. However, aromatherapy could not be considered an effective treatment to manage nervousness [30]. The other study concluded that aromatherapy could serve as an effective therapeutic for the relief from depressive symptoms in different individuals. In particular, aromatherapy massage appeared to be more effective than inhalation aromatherapy [31].

\subsection{Fatigue}

Twenty patients were enrolled in a single-arm trial to investigate the effectiveness of a combined modality treatment consisting of aromatherapy, foot soak, and reflexology on fatigue. Cancer Fatigue Scale (CFS) scores improved significantly after the treatment $(p<0.001)$, and no side effects were reported [32].

Another trial involving 105 hemodialysis patients showed that aromatherapy with lavender essential oil could decrease the level of fatigue compared to Benson relaxation techniques [33]. Another trial, again involving hemodialysis patients, reported that aromatherapy prepared with sweet orange and lavender oil could increase the quality of sleep and decrease fatigue levels [34].

\subsection{Insomnia}

Soden et al. reported, in a three-armed randomized trial (aromatherapy massage, massage with an inert carrier oil, and no massage), no significant differences in the mean Verran and SnyderHalpern Sleep Scale (VSH) from baseline to the final assessment. A direct comparison of the groups showed a difference between control and intervention groups; however, no differences between massage alone and aromatherapy massage were reported [5]. Moreover, in the trial by Stinger et al. that relied on aroma sticks, 31 patients received an aroma stick, mainly because of insomnia. Twenty-two (71\%) patients found them useful, whereas 8 (26\%) patients did not. One patient did not use it [12]. A meta-analysis of 12 studies revealed that the use of aromatherapy was effective in improving the quality of sleep. Here, the subgroup analysis revealed that inhalation aromatherapy was more effective than massage therapy. Thus, readily available aromatherapies appeared to be effective by promoting sleep [35].

Another study on 50 patients with newly diagnosed acute leukemia, who had been hospitalized to receive their initial four weeks of intensive induction chemotherapy, found aromatherapy as a viable intervention for improving insomnia and other symptoms commonly experienced by patients with acute leukemia [36].

\section{Conclusion}

The present review shows that aromatherapy has been widely studied in recent decades to treat several symptoms. However, few studies relate to a specific palliative need, and most are of low quality, with low patient numbers and often conducted as a one-armed trial. Other potential limitations or flaws include that interventions were not administered under the optimal situation. For example, massage was given in the morning, although the study was designed with a focus on sleep [9]. Furthermore, most studies used parent-evaluated questionnaires, such as HADS and the Rotterdam Symptom Checklist (RSCL), which capture different symptoms. Therefore, most of the surveys focused on pain, anxiety, and depression. 
A randomized controlled study on constipation yielded good, significant results, showing that aromatherapy massage was superior to massage alone [15]. The other symptoms were mostly evaluated in single-arm studies with no comparison arm. Although few studies could show positive results to a certain extent, the question of whether aromatherapy is useful remains unanswered. For example, Hsu et al. inferred that the improvement in the intervention group was primarily caused by the intervention itself rather than by aromatherapy [19]. It can be assumed that the touch itself leads to an improved relationship between patients and, thus, to an improvement in several symptoms in patients suffering from deadly diseases. Similar results were shown by Senterovich et al. in a retrospective chart review in a geriatric palliative care unit [37].

In the Cochrane database of systematic reviews, Fellowes et al. reported contradictory evidence for any additional benefit on anxiety conferred by the addition of aromatherapy [38]. In the update by Shin et al. (2016), only two studies compared massage to massage with aromatherapy. It was not possible to assess the effect of adding aromatherapy to massage on relief from pain; psychological symptoms, including anxiety and depression; physical symptom distress; or quality of life [39]. Therefore, the question of whether to include aromatherapy to actual interventions in palliative care remains unresolved. Although aromatherapy appears to be safe, its adverse effects have been described by several studies. For example, Candy et al. reported, in a systematic review, isolated allergic reactions appearing as rash, albeit rarely [4]. Similarly, other authors selected allergic respiratory diseases as exclusion criteria [6]. Furthermore, the results reported by different studies in favor of aromatherapy are inconsistent. Characteristics and results differed across interventions and even in the same intervention, as shown by Coelho et al. in a scoping review [40].

However, the present review also describes the proven efficacy of aromatherapy in several fields outside that of palliative care, yet related to relevant topics of palliative care. Certainly, the findings cannot be transferred directly to the palliative setting, as the background and causes of symptoms in a palliative situation could have different aspects. As it could be difficult to conduct trials in a palliative setting, it may be justified to transfer some findings to a palliative setting because patients may benefit from this readily available, cost-effective, efficacious, and safe treatment approach. Thus, the use of aromatherapy appears justified. Nevertheless, further highquality studies are necessary to support the benefits of aromatherapy.

\section{Author Contributions}

Heidrun Männle: Original idea, planning, and input of the manuscript. Data collection. Analysis and interpretation of data. Writing the manuscript.

Birthe Osorio: Reading/commenting/editing of manuscript. Critical revision of the manuscript.

Karsten Münstedt: Analysis and interpretation of data. Reading/commenting/editing of manuscript. Critical revision of the manuscript. Supervision of the manuscript. Training/education of authors. Mentoring of 1st author.

\section{Competing Interests}

The authors have declared that no competing interests exist. 


\section{References}

1. Bray F, Ferlay J, Soerjomataram I, Siegel RL, Torre LA, Jemal A. Global cancer statistics 2018: GLOBOCAN estimates of incidence and mortality worldwide for 36 cancers in 185 countries. CA Cancer J Clin. 2018; 68: 394-424.

2. Muecke R, Paul M, Conrad C, Stoll C, Muenstedt K, Micke O, et al. Complementary and alternative medicine in palliative care: A comparison of data from surveys among patients and professionals. Integr Cancer Ther. 2016; 15: 10-16.

3. Steflitsch W. Aromatherapy-from traditional and scientific evidence into clinical practice. Dtsch Med Wochenschr. 2017; 142: 1936-1942.

4. Candy B, Armstrong M, Flemming K, Kupeli N, Stone P, Vickerstaff V, et al. The effectiveness of aromatherapy, massage and reflexology in people with palliative care needs: A systematic review. Palliat Med. 2020; 34: 179-194.

5. Soden K, Vincent K, Craske S, Lucas C, Ashley S. A randomized controlled trial of aromatherapy massage in a hospice setting. Palliat Med. 2004; 18: 87-92.

6. Weaver MS, Robinson J, Wichman C. Aromatherapy improves nausea, pain, and mood for patients receiving pediatric palliative care symptom-based consults: A pilot design trial. Palliat Support Care. 2020;18: 158-163.

7. Berger $L$, Tavares $M$, Berger $B$. A Canadian experience of integrating complementary therapy in a hospital palliative care unit. J Palliat Med. 2013; 16: 1294-1298.

8. Abdelhakim AM, Hussein AS, Doheim MF, Sayed AK. The Effect of inhalation aromatherapy in patients undergoing cardiac surgery: A systematic review and meta-analysis of randomized controlled trials. Complement Ther Med. 2019: 48: 102256.

9. Lee MS, Lee HW, Khalil M, Lim HS, Lim HJ. Aromatherapy for managing pain in primary dysmenorrhea: A systematic review of randomized placebo-controlled trials. J Clin Med. 2018; 7: E434.

10. Chen SF, Wang $\mathrm{CH}$, Chan PT, Chiang HW, Hu TM, Tam KW, et al. Labour pain control by aromatherapy: A meta-analysis of randomised controlled trials. Women Birth. 2019; 32: 327335.

11. Lakhan SE, Sheafer H, Tepper D. The effectiveness of aromatherapy in reducing pain: $A$ systematic review and meta-analysis. Pain Res Treat. 2016; 2016: 8158693.

12. Stringer J, Donald G. Aromasticks in cancer care: An innovation not to be sniffed at. Complement Ther Clin Pract. 2011; 17: 116-121.

13. Asay K, Olson C, Donnelly J, Perlman E. The use of aromatherapy in postoperative nausea and vomiting: A systematic review. J Perianesth Nurs. 2019; 34: 502-516.

14. Ozgoli G, Naz MSG. Effects of complementary medicine on nausea and vomiting in pregnancy: A systematic review. Int J Prev Med. 2018; 9: 75.

15. Lai $T$, Cheung $M$, Lo $C, N g ~ K$, Fung $Y$, Tong $M$, et al. Effectiveness of aroma massage on advanced cancer patients with constipation: A pilot study. Complement Ther Clin Pract. 2011; 17: 37-43.

16. Preece J. Introducing abdominal massage in palliative care for the relief of constipation. Complement Ther Nurs Midwifery. 2002; 8: 101-105. 
17. Miyoung C, Euysoon C. A comparison between effects of aroma massage and meridian massage on constipation and stress in women college students. J Korean Acad Nurs. 2011; 41: 26-35.

18. Nam MJ, Bang YI, Kim TI. Effects of abdominal meridian massage with aroma oils on relief of constipation among hospitalized children with brain related disabilities. J Korean Acad Nurs. 2013; 43: 247-255.

19. Hsu CH, Chi CC, Chen PS, Wang SH, Tung TH, Wu SC. The effects of aromatherapy massage on improvement of anxiety among patients receiving palliative care: A systematic review of randomized controlled trials. Medicine. 2019; 98: e14720.

20. Wilkinson S. Aromatherapy and massage in palliative care. Int J Palliat Nurs. 1995; 1: 21-30.

21. Kyle G. Evaluating the effectiveness of aromatherapy in reducing levels of anxiety in palliative care patients: Results of a pilot study. Complement Ther Clin Pract. 2006; 12: 148-155.

22. Wilkinson S, Aldridge J, Salmon I, Cain E, Wilson B. An evaluation of aromatherapy massage in palliative care. Palliat Med. 1999; 13: 409-417.

23. Wilkinson SM, Love SB, Westcombe AM, Gambles MA, Burgess CC, Cargill A, et al. Effectiveness of aromatherapy massage in the management of anxiety and depression in patients with cancer: A multicenter randomized controlled trial. J Clin Oncol. 2007; 25: 532539.

24. Barati F, Nasiri A, Akbari N, Sharifzadeh G. The effect of aromatherapy on anxiety in patients. Nephrourol Mon. 2016; 8: e38347.

25. Serfaty M, Wilkinson S, Freeman C, Mannix K, King M. The ToT study: Helping with Touch or Talk (ToT): A pilot randomised controlled trial to examine the clinical effectiveness of aromatherapy massage versus cognitive behaviour therapy for emotional distress in patients in cancer/palliative care. Psychooncology. 2012; 21: 563-569.

26. Hadfield $\mathrm{N}$. The role of aromatherapy massage in reducing anxiety in patients with malignant brain tumours. Int J Palliat Nurs. 2001; 7: 279-285.

27. Kite S, Maher E, Anderson K, Young T, Young J, Wood J, et al. Development of an aromatherapy service at a cancer centre. Palliat Med. 1998; 12: 171-180.

28. Kang HJ, Nam ES, Lee Y, Kim M. How Strong is the Evidence for the Anxiolytic Efficacy of Lavender? Systematic review and meta-analysis of randomized controlled trials. Asian Nurs Res. 2019; 13: 295-305.

29. Thrane S, Hsieh K, Donahue P, Tan A, Exline MC, Balas MC. Could complementary health approaches improve the symptom experience and outcomes of critically ill adults? A systematic review of randomized controlled trials. Complement Ther Med. 2019; 47: 102166.

30. Babakhanian M, Ghazanfarpour M, Kargarfard L, Roozbeh N, Darvish L, Khadivzadeh T, et al. Effect of aromatherapy on the treatment of psychological symptoms in postmenopausal and elderly women: A systematic review and meta-analysis. J Menopausal Med. 2018; 24: 127-132.

31. Sánchez-Vidaña DI, Ngai SPC, He W, Chow JKW, Lau BWM, Tsang HWH. The effectiveness of aromatherapy for depressive symptoms: A systematic review. Evid Based Complement Alternat Med. 2017; 2017: 5869315.

32. Kohara H, Miyauchi T, Suehiro Y, Ueoka H, Takeyama H, Morita T. Combined modality treatment of aromatherapy, footsoak, and reflexology relieves fatigue in patients with cancer. J Palliat Med. 2004; 7: 791-796. 
33. Hassanzadeh M, Kiani F, Bouya S, Zarei M. Comparing the effects of relaxation technique and inhalation aromatherapy on fatigue in patients undergoing hemodialysis. Complement Ther Clin Pract. 2018; 31: 210-214.

34. Muz G, Taşcı S. Effect of aromatherapy via inhalation on the sleep quality and fatigue level in people undergoing hemodialysis. Appl Nurs Res. 2017; 37: 28-35.

35. Hwang $E$, Shin $\mathrm{S}$. The effects of aromatherapy on sleep improvement: A systematic literature review and meta-analysis. J Altern Complement Med. 2015; 21: 61-68.

36. Blackburn L, Achor S, Allen B, Bauchmire N, Dunnington D, Klisovic RB, et al. The effect of aromatherapy on insomnia and other common symptoms among patients with acute leukemia. Oncol Nurs Forum. 2017: 44: E185-E193.

37. Senderovich H, Ip ML, Berall A, Karuza J, Gordon M, Binns M, et al. Therapeutic Touch ${ }^{\circledR}$ in a geriatric Palliative Care Unit-A retrospective review. Complement Ther Clin Pract. 2016; 24: 134-138.

38. Fellowes D, Barnes K, Wilkinson SS. Aromatherapy and massage for symptom relief in patients with cancer. Cochrane Database Syst Rev. 2004: CD002287.

39. Shin ES, Seo KH, Lee SH, Jang JE, Jung YM, Kim MJ, et al. Massage with or without aromatherapy for symptom relief in people with cancer. Cochrane Database Syst Rev. 2016: CD009873.

40. Coelho A, Parola V, Cardoso D, Bravo ME, Apóstolo J. Use of non-pharmacological interventions for comforting patients in palliative care: A scoping review. JBI Database System Rev Implement Rep. 2017; 15: 1867-1904.

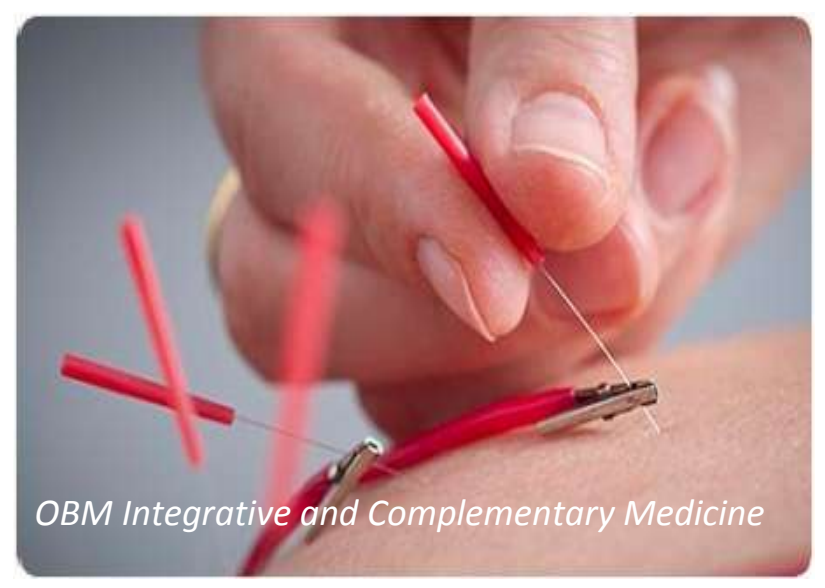

Enjoy OBM Integrative and Complementary Medicine by:

1. Submitting a manuscript

2. Joining in volunteer reviewer bank

3. Joining Editorial Board

4. Guest editing a special issue

For more details, please visit: http://www.lidsen.com/journals/icm 